\title{
Possible Dynamics of Polymer Chains by Means of a Ricatti's Procedure - an Exploitation for Drug Release at Large Time Intervals
}

\begin{abstract}
BOGDAN MIHAIL COBZEANU ${ }^{1}$, STEFAN IRIMICIUC², DORIN VAIDEANU ${ }^{2}$, ALEXANDRU GRIGOROVICI ${ }^{3 *}$, OVIDIU POPA
${ }^{1}$ Grigore T. Popa University of Medicine and Pharmacy of lasi, ENT Department, 16 Universitatii Str., 700115, Iasi, Romania

${ }^{2}$ Al. I. Cuza University of lasi, Faculty of Physics, 11 Carol I Blvd., 700506, Iasi, Romania

${ }^{3}$ Grigore T. Popa University of Medicine and Pharmacy of laoi, Department of Surgery, 16 Universitatii Str., 700115, iasi, Romania ${ }^{4}$ Grigore T. Popa University of Medicine and Pharmacy of lasi, Department of Emergency Medicine, 16 Universitatii Str., 700115, lasi, Romania
\end{abstract}

In this paper, we propose an analyzis method for the dynamics of polymer chains, based on a Ricatti procedure induced by possible correlations through the compatibility of the thermal radiation-classical damped oscillator dynamics. Then, the quantification (radiation frecquency is directly proportional to the color temperature) and the erasure of one bit of information becomes a natural processes induced by the system structure itself. The theory has implications in the dynamics of biological structures (arhitecture of tumors, information on tumor growth and angiogenesis, etc.) in the form of Resonant Recognition Model.

Keywords: damped oscillations, polymer chains dynamics, Riccati's equation

Recent progress in manipulating atomic and condensed matter systems has instigated a surge of interest in nonequilibrium physics, including many-body dynamics of trapped ultracold atoms and ions, near-field radiative heat transfer, and quantum friction. Under most circumstances the complexity of such nonequilibrium systems requires a number of approximations to make theoretical descriptions tractable. In particular, it is often assumed that spatially separated components of a system thermalize with their immediate surroundings, although the global state of the system is out of equilibrium. This powerful assumption reduces the complexity of nonequilibrium systems to the local application of well-founded equilibrium concepts. While this technique appears to be consistent for the description of some phenomena, itfails for quantum friction by underestimating by approximately $80 \%$ the magnitude of the drag force [1].

A striking feature of the quantum mechanical interaction between atoms or molecules and the electromagnetic field is the existence of dispersion forces such as Casimir-Polder and van der Waals forces [2]. In such a framework, the quantum paths in imaginary time (between 0 and $\beta=1$ / kT, where k is the Boltmann's constantand $T$ the absolute temperature) is equivalent, in classical terms, to polymer chains that form closed loops of length $\beta$. These polymer chains are in a state of random walk and the pair correlation function for points along each of them can be evaluated [3].

In this context, in the present paper, similar results with those from [3] are obtained by means of a Ricatti's procedure induced by possible correlations through the compatibility of the thermal radiation-classical damped oscillator dynamics. Thus, an analyzis method for the dynamics of polymeric chains is generated.

\section{Experimental part}

Hydrogels based on polymers (high and medium molecular weight chitosan, poly(vinyl alcohol), glutaraldehyde), cross-linked with sodium sulphate or sodium tripolyphosphate were prepared following the method detailed in [4]. The hydrogels composition was modified in order to optimize of their properties, in terms of mechanical stability and drug release. The hydrogels were loaded with calcein and the amount of released drug was monitorized, observing for large time intervals a decrease of released calcein [4].

This evolution could not be explained by the classical theories of drug release, based on diffusion, due to the high number of interconnected variables involved, so that we have taken into consideration the development of a new theoretical model.

\section{Theoretical method}

Let us consider the second order differential equation characterizing a classical damped harmonic oscillator:

$$
M q+2 R q+K q=0
$$

with obvious notation for first and second derivatives of the relevant coordinate $q$ in relation to time $t$. In relation (1) $M$ is the inertial mass, $R$ is the damping coefficient and $K$ is the elastic coefficient. Rewritten in the form:

$$
\dot{p}=-\frac{2 R}{M} p-\frac{K}{M} q \quad \dot{q}=p
$$

these equations induce a two-dimensional manifold of phase space type $(p, q)$ in which $p$ correspond to momentum type variable and $q$ to position type variable. The second equation (2) corresponds to the momentum definition. The equations (2) do not representa Hamiltonian system, since the associated matrix is not an involution [ 5 , 6]. The statement becomes clearer if we put our system in its matrix form:

$$
\left(\begin{array}{l}
\dot{p} \\
\dot{q}
\end{array}\right)=\left(\begin{array}{cc}
-2 \frac{R}{M} & -\frac{K}{M} \\
1 & 0
\end{array}\right)\left(\begin{array}{l}
p \\
q
\end{array}\right)
$$

As long as $M, R, K$ have constant values, this matrix equation, written in an equivalent form evidences the position of the energy and thus of the Hamiltonian. Indeed, from equation (3) we can obtain:

*email: alexandrugrigorovici@yahoo.com 

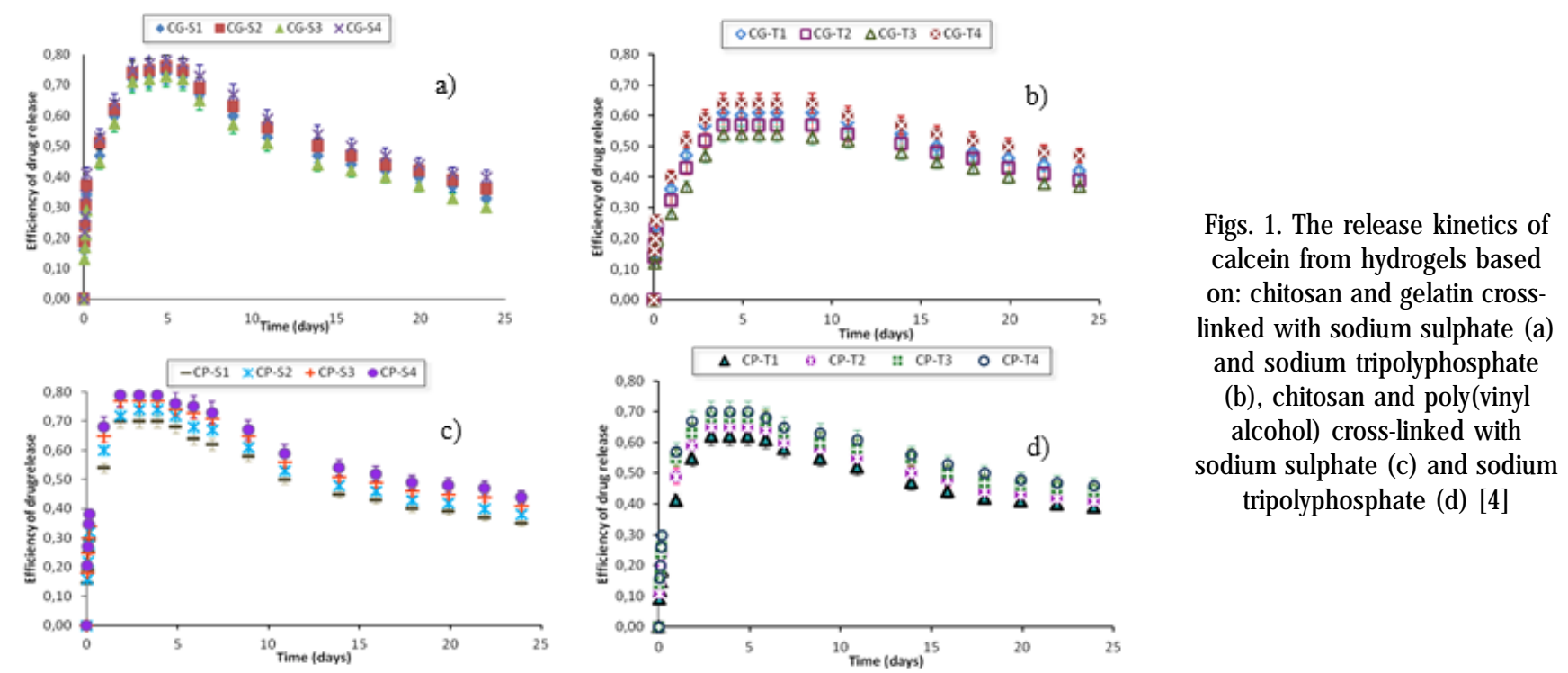

$$
\frac{1}{2} M(p \dot{q}-q \dot{p})=\frac{1}{2}\left(M p^{2}+2 R p q+K q^{2}\right)
$$

which proves that the energy in its quadratic form (the right part of equation (4)) is the variation rate of the physical action represented by the elementary area from the phase space. We would like to showcase here is the fact that the energy does not have to obey the conservation laws in order to act like a variation rate for the physical action. We now ask what could be the conservation law, if it exists? To give an adequate answer we first observe that the equation (4) can written as a Riccati type differential equation (on the Riccati's procedure, $[5,6]$ ):

$$
\phi+\phi^{2}+2 \mu \phi+\omega_{0}^{2}=0, \phi=\frac{p}{q}, \mu=\frac{R}{M}, \omega_{0}^{2}=\frac{K}{M}
$$

Furthermore, let us note that the Riccati type equation (5) is always represents a Hamiltonian system describing the harmonic oscillator type dynamics:

$$
\left(\begin{array}{l}
\dot{p} \\
\dot{q}
\end{array}\right)=\left(\begin{array}{cc}
-\frac{R}{M} & -\frac{K}{M} \\
1 & \frac{R}{M}
\end{array}\right)\left(\begin{array}{l}
p \\
q
\end{array}\right)
$$

This is a general characteristic describing the Riccati type equations and the Hamiltonian's dynamic $[5,6]$. Equation (4) can be obtained also by building from equations (6) the differential 1-form of the elementaryarea from the phase space for harmonic oscillator type dynamics. Regarding the equation (5), itcan be integrated by specifying the fact that the energy does not conserve anymore, but we find that another, more complicated dynamics variable will be conserved [7]:

$$
\frac{1}{2}\left(M p^{2}+2 R p q+K q^{2}\right) \cdot \exp \left\{\frac{2 R}{\sqrt{M K-R^{2}}} \arctan \left(\frac{M p+R q}{q \sqrt{M K-R^{2}}}\right)\right\}=\text { const }
$$

It results that the energy is conserved in a classical meaning if $\mathrm{R}$ becomes null or if the movement in the phase space is characterized by the line passing though origin, having the slope defined by the ratio between $\mathrm{R}$ and $M$. Moreover, the statistical character of energy reveals. Indeed, equation (7) can be written as:

$$
F(r, w)=\frac{K q^{2}}{\text { const. }}=\frac{1}{1+2 r w+w^{2}} \exp \left[\frac{2 r}{\left(1-r^{2}\right)^{1 / 2}} \arctan \frac{w\left(1-r^{2}\right)^{1 / 2}}{1-r w}\right],
$$

$$
w^{2}=\frac{M p^{2}}{K q^{2}}, r^{2}=\frac{R}{M}
$$

that explicitly shows that the potential energy, up to an arbitrary constant, depends in an effective manner on the ratio of kinetic and potential energy of the local oscillator. This energy can be correlated with an exponential factor (Boltzmann factor) that can play the role of partition function on a particular ensemble of harmonic oscillators of the same frequency (Planck's oscillators) in the form [8]:

$$
e^{-\beta \varepsilon_{0}}=\frac{1}{1+2 r w+w^{2}} \exp \left[\frac{2 r}{\left(1-r^{2}\right)^{1 / 2}} \arctan \frac{w\left(1-r^{2}\right)^{r / 2}}{1-r w}\right]
$$

The similarity of the equations (8) and (9) shows that the ratio of the two energies of the oscillators is in fact a statistical variable through which they are determined. In such conjecture, the left side of this equation represents a thermal ensemble of energy $\varepsilon_{\text {o }}$, with the average $\beta$. It is inconvenient that the right side also depends on $\varepsilon_{0}$. However, this dependence is realized through the ratio w, which allows us to affirm that a statistical interpretation depends on the content in $\varepsilon_{0}$ units of the thermal radiation field energy (fig. 2a, b).

If the energy density is of $\varepsilon_{0}$ order, then $w \approx 1$ and the right part of equation (9) depends only on the correlation coefficient $r$ of the two thermal radiation processes (one at high temperature and other at low temperature $[8,9])$ :

$$
e^{-\beta \varepsilon_{0}}=\frac{1}{2(1+r)} \exp \left[\frac{2 r}{\left(1-r^{2}\right)^{1 / 2}} \arctan \sqrt{\frac{1+r}{1-r}}\right]
$$

Relation (10) explicitly shows the relationship between the quanta and the statistical correlation of the two processes that represents thermal radiation. Moreover, it shows also the quanta value in the limit of the weak correlation, since we have:

$$
\varepsilon_{0}=k T \ln 2
$$

independent of any other consideration. Thus, this quanta and also frequency are directly proportional to color temperature. This fact was widely discussed by Louis de Broglie [10] that identify the action with entropy, in order to unify the physics in the so-called thermodynamics of the isolated particle. 


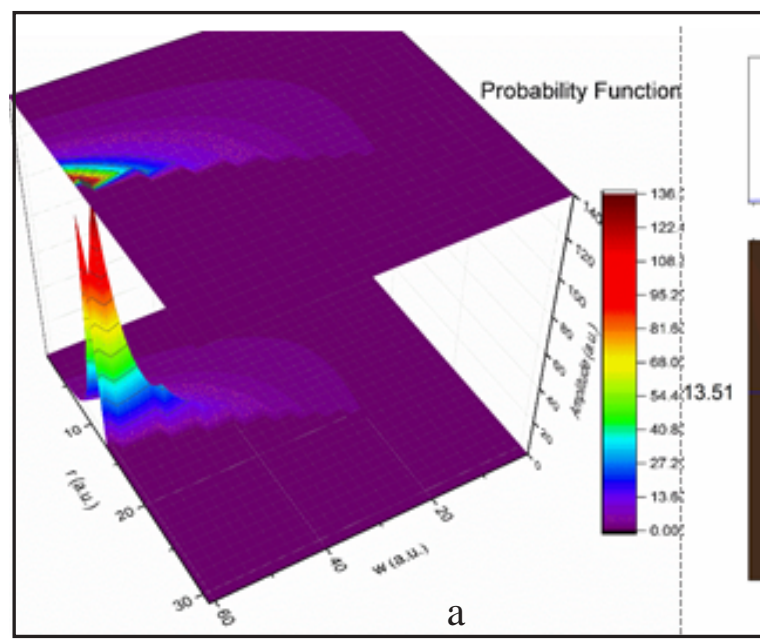

Let us remember that in 1961, Landauer [11] discussed the limitation of the efficiency of a computer imposed by physical laws. In particular, he argued that, according to the second law of thermodynamics, the erasure of one bit of information requires a minimal heat generation $k T \ln 2$, where $T$ is here the temperature at which one erases. Its arguments run as follows: since erasure is a logical function that does not have a single valued inverse it must be associated with physical irreversibility and, therefore, requires heat dissipation. A bit has one degree of freedom and so the heat dissipation should be of kT order. Now, since before erasure a bitcan be in any of the two possible states and after erasure it can only be in one state, this implies a change in information entropy of an amount $-k$ In 2 . In such a context, the idea that the polymer chains can presenta intrinsic pseudointelligence can be substantiated.

But, in the general case, when the content in $\varepsilon$ units of the thermal radiation field energy, as well as the correlation coefficient are arbitrary, it is important to note that the right part of the equation (9) is actually the generating function of a special class of Pollaczek polynoms. More precisely, we can write (9) in the form:

$$
e^{-\beta \varepsilon_{0}}=\sum_{n=0}^{\infty} P_{n}^{l}(r) w^{n}
$$

Their orthogonality relationship is given by:

$$
{ }_{-l}^{l} P_{n}^{a}(r) P_{m}^{a}(r) \rho(r) d r=\frac{\pi}{2} \frac{n+1}{n+a+1} \delta_{n m}
$$

with the weighting function $\rho$ :

$$
\begin{aligned}
& \rho(r)=\sin \theta \cdot e^{(2 \theta-\pi) \tan \theta}|\Gamma(1+i \tan \theta)|^{2}, \\
& r \equiv \cos \theta, \theta \in[0, \pi]
\end{aligned}
$$

where $\delta_{m n}$ is the Kronecker pseudotensor and $\Gamma$ is the first order Euler function, generalization of the factorial function. If we agree to interpret the energy as the energy of a photon, then the relation (12) is the starting point of a modern quantum state related to the coherence properties of the radiation.

Through such a procedure, the probability distribution (for example (14)) for the polymer amplitudes of a free harmonic oscillator can be found (equivalent procedures can be found in [3] and also, in its references). Moreover, new methodologies can be intuited, such as the fractal one [12-16] in order to analyze the polymer chains dynamics [17-18].

Additionally, we can say that an important variety of articles published in the Materiale Plastice journal have established optimal methods for obtaining different compounds (especially polymers), or stabilization and physico-chemical testing of high confidentiality [19-22].
Fig. 2a, b: Quantization procedure through correlation of all statistical ensembles associated with local oscillators; 3D dependences (a) and contour curves (b)

\section{Results and discussions}

Using a Ricatti's procedure, which implies possible correlations through the compatibility of the thermal radiation-classical damped oscillator dynamics, we have shown the following:

i) both potential energy of the damped oscillator and the distribution function of thermal radiation are defined by similar relations. In such a framework, the damping coefficient is directly related to the correlation coefficient of the distribution of the radiation, while the ratio between the kinetic and potential energies of the damped oscillator is directly related to energy distribution rate of thermal radiation; ii) if the correlation coefficient is null (i.e. in the absence of friction), a procedure of quantification was obtained. Then, the quanta value (radiation frequency is directly proportional to the color temperature) and the erasure of one bit of information results; iii) the theoretical model is validated by the similarity between the experimental curves (figs. 1) and the contour curves (fig. 2b)

\section{Conclusions}

Biological processes in any living organism are based on selective interactions between particular biopolymer chains. In most cases, these interactions involve and are driven by proteins which are the main conductors of any living process within the organism. The physical nature of these interactions is still not well known.

The work presented above aims to a whole new view to biopolymer interactions, based on the assumption thatthese interactions are electromagnetic in their nature.

So, we can consider that every biopolymer chain has its own specific resonating frequency where the same type of receptor biopolymer can be tuned to resonate specifically by means of a electromagnetic modality, i.e. electromagnetic resonance. By doing so (by tuning the receptors to the external energy source by means of electromagnetic resonance), a signal transduction response can invoked either by using a very little amount of ligands or without using any ligands at all, eventually obtaining the same end result therapeutically [23].

This new theory can incorporated in the Resonant Recognition Model (RRM) developed in [24].

The developed model is in good agreement with recent medical studies involving fractal geometry. Thus, itcan be used to describe the pathological architecture of tumors (see the distributions (14)) and moreover, to provide information about tumor growth and angiogenesis, mechanisms complementary to those obtained by modern molecular methods [25]. 


\section{References}

1.INTRAVAIA, F., BEHUNIN, R. O., HENKEL, C., BUSCH, K., DALVIT, D.A.R., Phys. Rev. Lett., 117, 2016, p. 100402

2.SCHEEL, S., BUHMANN, S.Y., Acta Phys. Slov., 58, 2008, p. 675

3.HOYE, J. S., BREVIK, I., Phys. A, 181, 1992, p. 413

4.BACAITA, E.S., CIOBANU, B.C., POPA, M., AGOP, M., DESBRIERES,

J., Phys. Chem. Chem. Phys., 16, 2014, p. 25896.

5.LIBERMANN, P., MARLE, CH., M., Symplectic Geometry and Analytical Mechanics, Springer, Netherlands, 1987.

6.ARNOLD, V. I., Mathematical Methods of Classical Mechanics, Springer Verlag, New York, USA, 1989.

7.DENMAN, H. H., Am. J. Phys., 36, 1968, p. 516

8.PLANCK, M., Planck's Original Papers in Quantum Physics, Wiley\&Sons Publishing, New York, 1972.

9.BORN, M., EINSTEIN, A., Light quantas, in Physics in my generation concept, Scientific and Encyclopedic Publishing House, Romania, 1969.

10.DE BROGLIE, L., La Thermodynamique de la particule isolee (ou Thermodynamique cachee des particules), Gauthier-Villars, Paris, 1964.

11.LANDAUER, R., IBM J. Res. Rev., 5, 1961, p. 183.

12.IORDACHE, D., PUSCA, S., TOMA, G., PAUN, V.P., STERIAN, A., MORARESCU, C., Lect Notes Comput SC, 3980, 2006, p.804.
13.AGOP, M., IOANNOU, P.D., NICA, P., RADU, C., ALEXANDRU, A., VIZUREANU, P., Mater. Trans., 45, 2004, p. 972

14.NEJ NERU, C., NICUTA, A., CONSTANTIN, B. MANEA, L.R., TEODORESCU, M., AGOP, M., J. Appl. Math., 137056, 2013.

15.NICULESCU, O., DIMITRIU, D. G., PAUN, V. P., MATASARU, P.D., SCURTU, D., AGOP, M., PHYSICS OF PLASMAS, 17, nr. 4, 2010, Article Number: 042305.

16.GURLUI, S., AGOP, M., STRAT, M., STRAT, G., BACAITA, S., Jpn. J. Appl. Phys., 44, 2005, p. 3253.

17.BACAITA, E.S., AGOP, M., Phys. Chem. Chem. Phys., 18, 2016, p. 21809.

18.RADU, V., BACAITA, S., ULINIUC, A., POPA, M., SUSANU, S., Mat. Plast., 50, 2013, p. 18.

19.PAUN, V. P., Mat. Plast., 44, no. 4, 2007, p. 393.

20.PAUN, V. P., Mat. Plast., 43, no.1, 2006, p. 57.

21.PAUN, V. P., Mat. Plast., 40, no. 1, 2003, p. 25.

22.PAUN, V. P., Mat. Plast., 40, no. 2, 2003, p. 81.

23.COSIC, I., PIROGOVA, E., Nonlinear Biomed. Phys., 1, nr. 1, 2007, p. 7.

24.COSIC, I., IEEE Trans. Biomed. Eng., 41, 1994, p. 1101. 25.BAISCH, J.W., JAIN, R.K., Cancer Res., 60, 2000, p. 3683.

Manuscript received: 18.03 .2017 